\title{
Satisfação corporal associada à gordura corporal e estado nutricional em jovens basquetebolistas
}

CDD. 20.ed. 302.222

796.32

http://dx.doi.org/10.1590/1807-55092015000200259

\author{
Leonardo de Sousa FORTES* \\ Dilson Borges RIBEIRO JUNIOR ${ }^{* *}$ \\ Santiago Tavares PAES ${ }^{* *}$ \\ Valter Paulo Neves MIRANDA*** \\ Maria Elisa Caputo FERREIRA ${ }^{* *}$
}

*Centro Acadêmico de Vitória, Universidade Federal de Pernambuco. **Faculdade de Educação Física e Desportos, Universidade Federal de Juiz de Fora.

\section{Resumo}

0 objetivo deste estudo foi comparar e verificar a possibilidade de associação entre a satisfação corporal (SC), classificações de estado nutricional (EN) e gordura corporal (GC) em jovens basquetebolistas masculinos. Participaram da pesquisa 93 adolescentes com idades de 10 a 16 anos. A SC foi avaliada pelo Body Shape Questionnaire. Foram coletadas massa corporal, estatura e dobras cutâneas para classificar o EN (kg/m²) e medir a GC, respectivamente. Realizaram-se os testes Ancova e Qui-quadrado no "software" SPSS 17.0. Identificaram-se diferenças estatisticamente significativas $(p<0,05)$ de SC entre classificações de EN e GC. Encontrou-se associação significativa $(p<0,05)$ entre SC e EN, assim como entre SC e GC $(p<0,05)$. Os resultados confirmaram diferenças e associação da SC com o EN e GC em jovens basquetebolistas.

PalaVRAS-CHAVE: Imagem corporal; Atletas; Basquetebol.

\section{Introdução}

O esporte infanto-juvenil, nos últimos anos, tem apresentado peculiaridades que exigem do esportista, o máximo de rendimento atlético, às vezes, em idades relativamente precoces. Atletas têm sido cobrados por treinadores, patrocinadores e pais para otimizarem a cada dia seus desempenhos esportivos. Melhorar capacidades fisiológicas, biomecânicas e psicológicas torna-se essencial para se atingir uma boa performance na maioria das modalidades esportivas ${ }^{1-2}$. Alguns esportes possuem características intermitentes e de tomada de decisão, devendo o atleta, aperfeiçoar tanto capacidades físicas, quanto habilidades psicológicas, como é o caso do basquetebol ${ }^{3}$.

Por sua vez, o basquetebol é uma modalidade esportiva praticada em diversos países no mundo. Os basquetebolistas costumam apresentar elevadas estaturas. Isto ocorre devido ao processo de seleção para este esporte, já que é exigido que o atleta seja alto, além de apresentar impulsão vertical aprimorada ${ }^{4}$. Muitas vezes a estatura elevada apresenta relaçáo com os processos de maturação/crescimento, principalmente o pico de velocidade de crescimento em estatura (PVA). Estatura elevada está associada ao aumento da massa corporal, pois geralmente, estes indivíduos apresentam maior densidade mineral óssea ${ }^{4}$. No entanto, segundo Conti et al. ${ }^{5}$, elevados valores de massa corporal estáo relacionados à demasiada preocupaçáo com o corpo. A massa corporal acentuada, juntamente com as pressóes e exigências encontradas no âmbito esportivo, podem gerar depreciação com o próprio corpo no atleta 5 .

Preocupaçôes e depreciaçôes com o peso e a aparência física são atribuídas ao conceito de insatisfação corporal $^{6-7}$. A prevalência deste sentimento afetivo na população adolescente gira em torno de $60 \% \%^{6,8-9}$, apresentando menores índices no sexo masculino ${ }^{10}$. Entretanto, a ocorrência deste fenômeno no público de atletas parece ser menor ${ }^{2,11}$, havendo ainda inconsistência da literatura a respeito desta prevalência ${ }^{12-13}$, principalmente entre os meninos ${ }^{14}$. Sabe-se que a insatisfaçáo corporal pode proporcionar o desencadeamento de efeitos deletérios a saúde ${ }^{1,15}$. Autores 
argumentam que a insatisfação com o corpo é sintoma de primeira ordem no aparecimento de conduta alimentar anormal ${ }^{12,16}$, podendo esta, resultar em síndromes psicológicas como é o caso dos transtornos alimentares $(\mathrm{TAs})^{11,13,17}$. Vale ressaltar, ainda, que de acordo com a literatura científica ${ }^{5,18}$, os basquetebolistas do sexo masculino estão mais vulneráveis ao desencadeamento de TAs em comparação aos atletas de outras modalidades esportivas, o que justifica a realização de investigaçôes com este público.

Sexo feminino, faixa etária adolescente, etnia branca e os estratos mais altos da sociedade são apresentados pela literatura científica como possíveis fatores de risco no surgimento de insatisfação corporal $^{16,19}$. Além destes, a gordura corporal elevada e o estado nutricional inadequado também têm

\section{Método}

\section{Participantes}

Trata-se de um estudo transversal realizado no ano de $2011 \mathrm{com}$ jovens atletas do sexo masculino, vinculados a três clubes de basquetebol da cidade de Três Rios/RJ, com idade entre os 10 e 16 anos. Os sujeitos constituem inicialmente 105 indivíduos, inscritos e participantes de competiçôes na Federação de Basquetebol do estado do Rio de Janeiro (FBERJ). Para o cálculo da amostra, adotou-se percentual máximo de $15 \%$ para insatisfação corporal a partir dos achados de Fortes e Ferreira ${ }^{5}$ e Fortes et al. ${ }^{21}$, utilizando a equação de FieLD ${ }^{22}$, que considera erro relativo tolerável de cinco pontos percentuais e intervalo de confiança de $95 \%$, obtendo-se um tamanho amostral estimado de 69 adolescentes.

Para participar do estudo, os sujeitos deveriam apresentar o termo de consentimento livre e esclarecido (TCLE) assinado pelo responsável, treinar sistematicamente basquetebol pelo menos cinco vezes na semana com duração mínima de duas horas por sessão, além de ter participado de alguma competição no ano de 2011.

Desta forma, foram avaliados 99 indivíduos. No entanto, seis atletas foram excluídos da pesquisa por não responderem os questionários em sua totalidade ou não participarem das aferiçóes antropométricas. Portanto, a amostra final do estudo foi constituída por 93 jovens. sido associados à insatisfação corporal em adolescentes $^{6,8-9,20}$. Entretanto, grande parte dos estudos foram desenvolvidos somente com a população de escolares, geralmente utilizando o sexo feminino como amostra ${ }^{8-9}$. Por isso, a população de atletas adolescentes, principalmente do sexo masculino, não tem sido devidamente avaliada no que diz respeito a variáveis afetivas ou comportamentais. Vale destacar, ainda, que evidenciar se a satisfação corporal está ou não associada a composiçáo corporal em jovens basquetebolistas ajudaria treinadores na identificação do problema. Diante do exposto acima, o objetivo do presente estudo foi comparar e verificar a possibilidade de associação entre a satisfação corporal, as classificaçóes de estado nutricional e a gordura corporal em jovens basquetebolistas do sexo masculino.

\section{Instrumentos}

\section{Satisfação corporal}

Avaliou-se por intermédio do Body Shape Questionnaire (BSQ). Trata-se de uma escala autoreportada constituída por 34 perguntas que se propóem avaliar a preocupação com o peso e a forma física. O BSQ é uma escala do tipo likert com seis opçóes de resposta para cada questão (Nunca $=1$ até Sempre = 6). Ele classifica níveis de insatisfação a respeito do corpo, sendo: $<80$ pontos livre de insatisfação corporal, entre 80 e 110 leve insatisfação, entre 110 e 140 insatisfação moderada e pontuaçôes acima de 140 grave insatisfaçáo corporal, ou seja, quanto maior o escore, maior a insatisfação com o corpo. A versão utilizada foi validada para adolescentes brasileiro ${ }^{20} \mathrm{e}$ sua análise de consistência interna revelou alpha de Cronbach de 0,96 e coeficiente de correlação entre os escores do teste-reteste significativo. Calculouse a consistência interna para a presente amostra, identificando-se valor alpha de 0,87 .

\section{Antropometria}

Foram coletadas massa corporal, estatura e dobras cutâneas (subescapular e triciptal). Utilizaram-se os procedimentos descritos pela Internacional Society for Advancement for Kineanthropometry (ISAK) ${ }^{23}$. 
A massa corporal e a estatura foram mensuradas com os avaliados descalços e com o mínimo de roupa. Utilizou-se uma balança eletrônica portátil da marca Tanita, com precisão de $0,1 \mathrm{~kg}$, para a massa corporal, e um estadiômetro portátil, com precisão de 0,5 $\mathrm{cm}$, da marca Tonelli, para a estatura. Essas variáveis foram mensuradas uma única vez. O Índice de Massa Corporal (IMC) foi obtido pela razão entre massa corporal $(\mathrm{kg})$ dividido pelo quadrado da estatura $(\mathrm{m})$.

A classificação do estado nutricional foi obtida mediante os pontos de corte do IMC estabelecidos pela Organização Mundial de Saúde ${ }^{24}$, que os separa por intermédio do percentil $(5,85$ e 95) em quatro grupos contrastes segundo sexo e idade cronológica: baixo peso (percentil $\leq 5$ ), eutrófico (percentil $>5$ e $<85$ ), sobrepeso (percentil $\geq 85$ e $<95$ ) e obeso (percentil $\geq 95$ ).

As medidas das dobras cutâneas foram efetuadas em triplicata, de forma não consecutiva. Estas foram mensuradas com plicômetro da marca Lange com a precisão de $1 \mathrm{~mm}$. Estas medidas foram realizadas do lado direito do corpo. A medição da dobra cutânea do tríceps foi feita na parte posterior do braço, no ponto médio entre o processo acromial e o olécrano. A medição da dobra cutânea subescapular foi realizada $2 \mathrm{~cm}$ abaixo do ângulo inferior da escapula a uma inclinação de $45^{\circ}$ em relação ao lado do corpo.

Para os cálculos da percentagem de gordura (\%G), utilizou-se a equação de predição proposta por Slaughter et al. ${ }^{25}$. Determinou-se a gordura corporal pelo percentual de gordura. A classificação dessa variável foi atribuída pelos pontos de corte estabelecidos por LoHMAN ${ }^{26}$, que levam em consideração o sexo do sujeito. Juntaram-se as classificações "muito baixo" e "baixo" denominando o grupo "baixo"; "moderadamente alto", "alto" e "muito alto" denotando o grupo "alto".

\section{Procedimentos}

Os procedimentos foram realizados no horário do treinamento das equipes em dois dias subsequentes. Todos os clubes disponibilizaram salas e ambientes para proceder tais avaliações.

As coletas do questionário foram realizadas por apenas um pesquisador. Este ficou responsável pela aplicação destes instrumentos no primeiro momento. $\mathrm{O}$ segundo encontro destinou-se as aferições antropométricas (massa corporal, estatura e dobras cutâneas), cuja realização ficou restrita ao mesmo pesquisador com experiência neste tipo de avaliação. Salienta-se que as aferições antropométricas foram realizadas no período da tarde, mais especificamente, entre 15 e $17 \mathrm{~h}$ para todos os atletas.

No primeiro encontro aplicou-se o instrumento BSQ. O questionário foi entregue aos atletas que receberam a mesma orientação verbal. A orientação escrita dos procedimentos adequados constava no mesmo. Eventuais dúvidas foram esclarecidas pelo responsável pela aplicação deste instrumento. Os sujeitos do estudo não comunicavam entre si. Efetuou-se a distribuiçáo do questionário no momento que os atletas adentravam no ambiente (sala) e o preenchimento deste constituiu-se de caráter voluntário. Não houve limite de tempo para preenchê-lo.

No segundo momento os atletas foram conduzidos para aferição das variáveis antropométricas. A entrada dos atletas na sala de avaliação foi individualizada, permitindo-se outro sujeito adentrar no ambiente somente após seu colega de equipe retirar-se do recinto.

Todos os procedimentos somente foram realizados após aprovação do projeto no Comitê de Ética e Pesquisa em Seres Humanos da Universidade Federal de Juiz de Fora, recebendo parecer n. 232/2010, de acordo com a resolução 196 de 1996.

\section{Análise estatística}

Em razão do teste Kolmogorov-Smirnov não indicar violação paramétrica dos dados, utilizaramse medidas de tendência central (média e desvio padrão) para descrever as variáveis idade, IMC, percentual de gordura e satisfação corporal. Foi conduzida análise univariada de covariância (ANCOVA), utilizando idade como covariável, para comparar a satisfação corporal entre classificações de estado nutricional e gordura corporal. Conduziu-se o Qui-quadrado de Pearson para associar as variáveis dicotômicas do estudo (satisfação corporal, gordura corporal e estado nutricional). Para isso, as classificaçôes de insatisfação corporal do BSQ (leve, moderada e grave insatisfação) foram agrupadas em "insatisfeito". Todos os dados foram tratados no "software" SPSS versão 17.0, incutindo-se nível de significância de 5\%. 


\section{Resultados}

Os achados indicaram prevalência de $11 \%$ para a insatisfaçáo corporal. No tocante ao estado nutricional, os resultados apontaram 16\% dos basquetebolistas com baixo peso, $47 \%$ eutróficos, $19 \%$ e $18 \%$ com sobrepeso e obesidade, respectivamente. Concernente à gordura corporal, foram identificados
19\% com classificação "baixo", 51\% "normal" e os $30 \%$ restantes classificados como "alto".

Os dados descritivos (média e desvio padrão) das variáveis idade, IMC, percentual de gordura e satisfação corporal estão ilustrados na TABELA 1.
$B S Q=$ Body Shape Questionnaire. ap $<0,05$ em relação ao grupo "baixo peso"; ${ }^{\mathrm{b}} \mathrm{p}<0,05$ em relação ao grupo "eutrófico": ${ }^{d} p<0,05$ em relação ao grupo "obeso";

ep < 0,05 em relação ao grupo "baixo";

f $p<0,05$ em relação ao grupo "normal"; ${ }^{9} p<0,05$ em relação ao grupo "alto".

TABELA 1 - Idade, percentual de gordura, índice de massa corporal e satisfação corporal dos basquetebolistas avaliados.

\begin{tabular}{lc}
\hline Variável & Média (Desvio padrão) \\
\hline Idade & $14,91( \pm 2,00)$ \\
Percentual de gordura & $22,19( \pm 9,85)$ \\
Índice de Massa Corporal & $22,04( \pm 3,78)$ \\
BSQ & $68,98( \pm 26,60)$ \\
\hline
\end{tabular}

A TABELA 2 apresenta a comparação da satisfação corporal de acordo com o estado nutricional e a gordura corporal dos atletas de basquete. Atletas com baixo peso e/ou eutróficos demonstraram maior satisfação com o peso e a aparência física quando comparados aos obesos $(\mathrm{p}<0,05)$. Estes achados não se replicaram para atletas classificados com sobrepeso. Ademais, os basquetebolistas com baixa e média gordura corporal apresentaram maior satisfação com o corpo em relação a jovens com alto perfil de gordura corpórea $(\mathrm{p}<0,05)$.

TABELA 2 - Padrão da satisfação corporal (BSQ) segundo estado nutricional e gordura corporal dos basquetebolistas avaliados.

\begin{tabular}{lrcc}
\hline Variável & & n & BSQ \\
\hline Estado nutricional & & & \\
& Baixo peso & 15 & $62,00( \pm 7,00)^{\mathrm{d}}$ \\
& Eutrófico & 43 & $63,43( \pm 23,95)^{\mathrm{d}}$ \\
& Sobrepeso & 18 & $72,80( \pm 20,57)$ \\
Gordura corporal & Obeso & 17 & $108,17( \pm 24,03)^{\mathrm{a}, \mathrm{b}}$ \\
& & & \\
& Baixo & 18 & $50,66( \pm 11,50)^{\mathrm{g}}$ \\
& Normal & 28 & $57,20( \pm 6,23)^{\mathrm{g}}$ \\
& Alto & 47 & $76,98( \pm 4,24)^{\mathrm{e}, \mathrm{f}}$ \\
\hline
\end{tabular}

Encontrou-se associação significativa $(\mathrm{p}<0,05)$ entre satisfação corporal e estado nutricional (TABELA 3), assim como entre satisfação corporal e gordura corporal $(\mathrm{p}<0,05)$ (TABELA 4) em jovens basquetebolistas. Grande parte dos atletas $(57,14 \%)$ satisfeitos com sua aparência física estavam classificados como eutróficos a respeito de seu estado nutricional $(\mathrm{p}<0,05)$. Em relação à gordura corporal, todos os basquetebolistas $(33,3 \%)$ insatisfeitos com o corpo apresentavam alto perfil de gordura corporal $(\mathrm{p}<0,05)$. 
TABELA 3 - Frequência relativa de (in)satisfação corporal segundo estado nutricional dos basquetebolistas avaliados.

\begin{tabular}{lcccccc}
\hline & & Baixo peso & Eutrófico & Sobrepeso & Obeso & p valor \\
\cline { 3 - 7 } \multicolumn{2}{l}{ Insatisfação corporal } & & & & & \\
Insatisfeito & $28,62 \%$ & $0 \%$ & $11,11 \%$ & $7,9 \%$ & $9,52 \%$ & \\
Satisfeito & $71,38 \%$ & $7,9 \%$ & $57,14 \%$ & $4,76 \%$ & $1,58 \%$ & $\mathrm{p}<0,05^{*}$ \\
Total & $100 \%$ & $7,9 \%$ & $68,25 \%$ & $12,66 \%$ & $11,1 \%$ & \\
\hline
\end{tabular}

${ }^{*}$ Associação significativa pelo teste quiquadrado.

TABELA 4 - Frequência relativa de (in)satisfação corporal segundo gordura corporal dos basquetebolistas avaliados.

\begin{tabular}{lccccc}
\hline & & Baixo & Normal & Alto & p valor \\
\cline { 3 - 6 } \multicolumn{2}{l}{ Insatisfação corporal } & & & & \\
Insatisfeito & $33,33 \%$ & $0 \%$ & $0 \%$ & $33,33 \%$ & \\
Satisfeito & $66,67 \%$ & $12,69 \%$ & $28,57 \%$ & $25,39 \%$ & $\mathrm{p}<0,05^{*}$ \\
Total & $100 \%$ & $12,69 \%$ & $28,57 \%$ & $58,72 \%$ & \\
\hline
\end{tabular}

*Associação significativa pelo teste quiquadrado.

\section{Discussão}

O presente estudo teve como premissa comparar e verificar a possibilidade de associação entre a satisfação corporal, as classificaçôes de estado nutricional e a gordura corporal em jovens basquetebolistas do sexo masculino. As síndromes derivadas da excessiva preocupaçáo com o corpo estáo se convertendo numa verdadeira epidemia ${ }^{8,15}$. Entretanto, desejar uma imagem corporal perfeita não implica sofrer de uma doença mental, mas aumenta as possibilidades de que esta apareça, ${ }^{5,19}$. O ambiente atlético parece potencializar estes riscos ${ }^{3,13,27}$. A cultura esportiva para algumas modalidades coloca grande ênfase na magreza e em corpos com delineamento muscular perfeito ${ }^{28}$. No entanto, a genética não é favorável para se atingir esta estética para muitos destes sujeitos, o que faz com que cada vez mais os atletas se sintam incomodados com o peso e a aparência física. Atualmente, pesquisas no âmbito esportivo têm sido desenvolvidas associando fatores considerados de risco como possíveis desencadeadores de comportamentos inadequados (alimentação) $)^{3,13}$. Alguns achados apontam os traços da personalidade (perfeccionismo), a baixa autoestima e a depressão como variáveis negativas para o surgimento de tais comportamentos ${ }^{3,12}$. No entanto, raros estudos se propuseram avaliar sentimentos afetivos (insatisfação corporal) no público de atletas brasileiros ${ }^{2,529}$. Ademais, não se identificou pesquisa que tenha associado e comparado as classificaçóes antropométricas depreciadas na cultura ocidental (IMC e gordura corporal $)^{6}$ com a satisfação corporal nestes indivíduos.
Portanto, estima-se que este seja o primeiro estudo no Brasil a ser realizado com estas características.

Os principais achados da presente pesquisa foram: 1) encontrar menor satisfação corporal em basquetebolistas obesos e com alto percentual de gordura; 2) identificar associação da satisfação corporal com o estado nutricional e a gordura corporal. Percebeu-se que a maioria dos basquetebolistas satisfeitos com o corpo eram eutróficos $(57,14 \%)$ e todos os sujeitos insatisfeitos apresentaram alta gordura corporal (33,33\%).

Os resultados do presente estudo evidenciaram que atletas classificados como obesos por meio dos pontos de corte do IMC eram mais insatisfeitos com o corpo do que basquetebolistas com baixo peso e eutróficos. Apesar de não encontrar-se diferença estatisticamente significativa nas pontuaçóes do BSQ de atletas com sobrepeso e sujeitos com estados nutricionais de baixo peso e eutrofia, identificou-se pequena tendência. Indivíduos com sobrepeso/ obesidade apresentaram maior insatisfação com o peso e a aparência física quando comparados aos atletas com menores valores de IMC. Parece que em atletas de basquete do sexo masculino, as diferenças de satisfaçáo corporal segundo o estado nutricional foram semelhantes aos achados descritos com amostras de escolares deste mesmo sexo ${ }^{6,10}$. Por outro lado, autores sugerem que indivíduos que praticam atividade física sistemática costumam apresentar maiores índices de satisfação corporal, independente do estado nutricional ${ }^{1,14,19}$. 
Remetendo-se a comparação das pontuaçôes do BSQ entre as classificações de gordura corporal, o presente estudo apresenta resultados interessantes. Evidenciou-se que atletas com elevada composição de gordura corporal eram mais insatisfeitos com o corpo quando comparados aos esportistas inseridos nas classificaçôes de gordura corporal "baixo" e "normal". Ademais, assim como ocorreu com o estado nutricional, também se identificou tendência nas pontuaçôes de satisfação corporal entre as classificações da gordura corporal. Apesar da não significância estatística, percebeu-se que basquetebolistas com composiçáo de gordura "normal" apresentaram maiores pontuaçóes do BSQ em relação ao grupo "baixo". FilAire et al. ${ }^{13}$ encontraram achados semelhantes aos do presente manuscrito, pois identificaram que pontuaçóes em subescalas de insatisfação corporal eram significativamente maiores em ciclistas e judocas que apresentavam maior perfil de gordura corporal.

Em relaçáo à satisfaçáo corporal e o estado nutricional, o teste qui-quadrado mostrou associação significativa entre estas variáveis dicotômicas em basquetebolistas adolescentes. Parece que as classificaçôes inadequadas de estado nutricional (sobrepeso/obeso) influenciaram negativamente a prevalência de satisfaçáo corporal em jovens atletas de basquetebol. Além disso, grande parte dos esportistas eutróficos mostraram satisfação com seus corpos. Entretanto, estudos têm apontado hipóteses residuais. Krentz e WarchSburger ${ }^{28}$ salientam que valores elevados de IMC náo remetem necessariamente a preocupaçôes corporais em atletas de esportes de equipe com bola, como é o caso do basquetebol. Por outro lado, Torstveit e Sundgot-Borgen ${ }^{1}$ afirmam que o nível competitivo, juntamente com as pressóes de treinadores no anseio por melhores resultados são os principais agentes negativos para despertar depreciaçóes com a aparência no atleta. Talvez, a satisfação com o corpo nestes jovens esteja mais atrelada ao rendimento esportivo. Pesquisas mostram que quando o atleta não consegue otimizar seu rendimento atlético, este apresenta-se insatisfeito com sua aparência física, independente de suas características morfológicas ${ }^{11,29}$.

Agora remetendo a relação entre satisfação e gordura corporal, encontrou-se associação significativa entre estas variáveis. Todos os atletas insatisfeitos com o corpo estavam inseridos no grupo "alto" de gordura corporal. Estes resultados corroboram achados de outros autores ${ }^{13}$, inclusive em pesquisas envolvendo escolares ${ }^{8-9}$. KRentz e WARCHSBURGER $^{28}$ explicam que a gordura corporal é a principal característica morfológica depreciada em atletas, pois os esportistas associam o baixo percentual de gordura com o elevado rendimento esportivo, independente da modalidade praticada.

O presente estudo procurou preencher uma lacuna do conhecimento existente na literatura. Entretanto, esta pesquisa apresentou limitaçóes que merecem ser destacadas. A principal foi utilizar instrumento autoaplicável, pois indivíduos podem não responder com fidedignidade a esta ferramenta de pesquisa. Portanto, os resultados podem não refletir a realidade do contexto avaliado, visto que o resultado final é fruto de respostas subjetivas. Acrescentando, trata-se de uma pesquisa com delineamento transversal e por este motivo, possui baixa capacidade para estabelecer relaçóes de causa e efeito, tendo em vista que a coleta de dados sobre fatores de risco e o desfecho foi realizada simultaneamente e, portanto, dificulta o conhecimento da relaçáo temporal existente entre eles. Além disso, autores não sugerem a utilização do IMC como critério de corte para sobrepeso/obesidade em atletas ${ }^{1}$. De qualquer forma, este estudo acrescenta à literatura algumas questóes relevantes sobre a (in)satisfação corporal em basquetebolistas adolescentes brasileiros do sexo masculino que, até então, não havia sido explorada.

Diante das reflexôes descritas acima, os resultados do presente estudo confirmam a existência de associação da satisfação corporal com o estado nutricional e a gordura corporal em jovens basquetebolistas, além de identificar diferenças de pontuaçóes do BSQ entre grupos contrastantes destas variáveis dicotômicas.

Do ponto de vista acadêmico, os resultados desta pesquisa indicam a necessidade de se discutir sobre os padróes corporais vigentes. De acordo com Fortes et al. ${ }^{21}$, até mesmo os atletas parecem se preocupar com a gordura corporal, talvez, mais do que o desempenho esportivo. Assim, os basquetebolistas com sobrepeso ou gordura corporal avantajada podem gerar sentimentos depreciativos com o corpo em razão do que a mídia costuma preconizar, mais especificamente, corpos magros e com musculatura bem delineada ${ }^{10}$. Neste sentido, BAUM ${ }^{17}$ ressalta que embora os atletas devam se preocupar em maximizar o desempenho esportivo, é possível a estética corporal ser o atributo mais importante para este público.

Ademais, os achados da presente pesquisa evidenciam a necessidade de acompanhamento de psicólogos no processo de ensino-aprendizagem-treinamento de jovens basquetebolistas do sexo masculino de Três Rios/ RJ. Talvez, aconselhamentos destes profissionais possam prevenir o surgimento de síndromes psicológicas em 
esportistas com idades relativamente precoces. Além disso, faz-se necessário que treinadores de equipes de jovens tenham conhecimento a respeito de fatores de risco associados a variáveis afetivas e comportamentais, a fim de minimizar possíveis efeitos deletérios a saúde psicológica dos atletas.

Programas de avaliação e orientação, tanto psicológica, quanto nutricional, são necessários no âmbito esportivo. Estas iniciativas devem ser desenvolvidas com o caráter de prevenção e detecção de doenças como os TAs em atletas.

Sugere-se que sejam realizadas mais pesquisas nesta população com características semelhantes a este estudo, englobando outras modalidades esportivas e averiguando se o tipo de esporte exerce influência sobre variáveis afetivas.

\begin{abstract}
Body satisfaction associated to body fat and nutritional state in young basketball players

The objective of this study was to compare and verify the possibility of association between body satisfaction (IC), classifications nutritional status (EN) and body fat (BF) in young basketball players male. 63 teens aged 10 to 16 years participated in the survey. The IC was evaluated by through the Body Shape Questionnaire. We collected body mass, height and skinfolds to classification the EN $(\mathrm{kg} /$ $\mathrm{m}^{2}$ ) and measure BF, respectively. ANCOVA and chi-square tests were performed using SPSS 17.0. It was identified statistically significant differences $(p<0.05)$ between the $I C$ ratings $E N$ and $B F$. A significant association $(p<0.05)$ between IC and EN was found as well as between IC and BF $(p<0.05)$. The results confirmed the association of IC and differences with $\mathrm{EN}$ and $\mathrm{BF}$ on basketball.
\end{abstract}

KEY WoRDS: Body image; Athletes; Basketball.

\title{
Referências
}

1. Torstveit MK, J Sundgot-Borgen. The female athlete triad exists in both elite athletes and controls. Med Sci Sports Exerc. 2005;37:1449-59.

2. Vieira JLL, Amorim HZ, Vieira LF, Amorim AC, Rocha PGM. Distúrbios de atitudes alimentares e distorçáo da imagem corporal no contexto competitivo da ginástica rítmica. Rev Bras Med Esporte. 2009;15:410-4.

3. Schaal K, Tafflet M, Nassif H, et al. Psychological balance in high level athletes: gender-based differences and sportspecific patterns. PLoS One. 2011;6:1-9.

4. Malina R, Bouchard C, Bar-Or O. Crescimento, maturação e atividade física. São Paulo: Phorte; 2009.

5. Fortes LS, Ferreira MEC. Comparação da insatisfação corporal e do comportamento alimentar inadequado em atletas adolescentes de diferentes modalidades esportivas. Rev Bras Educ Fis Esporte. 2011;25:707-16.

6. Conti MA, Gambardella AMD, Frutuoso, MFP. Insatisfação com a imagem corporal em adolescentes e sua relação com a maturação sexual. Rev Bras Crescim Desenv Hum. 2005;15: 36-44.

7. Morgado FFR, Ferreira MEC. Escala de silhuetas bidimensionais: uma investigação acerca de sua aplicabilidade ao cego congênito. Rev Bras Cien Esporte. 2010;31: 61-73.

8. Martins CR, Pelegrini A, Matheus SC, Petroski EL. Insatisfação com a imagem corporal e a relação com estado nutricional, adiposidade corporal e sintomas de anorexia e bulimia em adolescentes. Rev Psiq Rio Grande do Sul. 2010;32:19-23.

9. Scherer FC, Martins CB, Pelegrini A, Matheus SC, Petroski EL. Imagem corporal em adolescentes: associação com a maturação sexual e sintomas de transtornos alimentares. Rev Bras Psiq. 2010;59:198-202.

10. Petroski, EL, Pelegrini A, Glaner MF. Insatisfação corporal em adolescentes rurais e urbanos. Motricidade. 2009;5: 13-25.

11. Perini TA, Vieira RS, Vigário OS, Oliveira GL, Ornellas JS, Oliveira FP. Transtorno do comportamento alimentar em atletas de elite de nado sincronizado. Rev Bras Med Esporte. 2009;15:54-57.

12. De Bruin AP, Oudejans RRD, Bakker FC. Dieting and body image in aesthetic sports: a comparison of dutch female gymnnasts and non-aesthetic sport partcipants. Psychol Sport Exerc. 2007;8:507-20. 
Fortes LS, et al.

13. Filaire E, Rouveix M, Pannafieux C, Ferrand C. Eating atitudes, perfectionism and body-esteem of elite male judoists and cyclists. J Sports Sci Med. 2007;6:50-7.

14. Hausenblas HA, Simons-Downs D. Comparison of body image between athletes and nonathletes: a aeta-analytic review. J Appl Sports Psychol. 2001;13:323-39.

15. Castro IRR, Levy RB, Cardoso LO, et al. Imagem corporal, estado nutricional e comportamento com relação ao peso entre adolescentes brasileiros. Cienc Saude Colet. 2010;15(Supl. 2):3099-108.

16. Sampei MA, Singulem DM, Novo NF, Juliano Y, Colugnati FAB. Atitudes Alimentares e imagem corporal em meninas adolescentes de ascendência nipônica e caucasiana em São Paulo (SP). J Ped. 2009;85:122-8.

17. Baum A. Eating disorders in the male athletes. Sports Med. 2006;36:1-6.

18. Bratland-Sanda S, Sundgot-Borgen J. Eating disorders in athletes: overview of prevalence risk factors and recommendations for prevention and treatment. Eur J Sport Sci. 2012;1:1-10.

19. Fortes LS, Miranda VPN, Amaral ACS, Ferreira MEC. Insatisfaçáo corporal de adolescentes atletas e não atletas. J Bras Psiq. 2011;60:309-14.

20. Conti MA, Cordás TA, Latorre MRDO. Estudo de validade e confiabilidade da versão brasileira do body shape questionnaire (bsq) para adolescentes. Rev Bras Saúde Mater Infant. 2009;9:331-8.

21. Fortes LS, Almeida SS, Ferreira MEC. Insatisfação corporal e maturação biológica em atletas do sexo masculino. Rev Bras Educ Fís Esporte. 2013;27:297-303.

22. Field A. Descobrindo a estatística usando o SPSS. Porto Alegre: Artmed; 2009.

23. The Internacional Society for Advancement for Kineanthropometry [homepage on the Internet]. Sidney: National Library of Australia [cited 2013 Jul 2013]. Available from: http://www.isakonline.com.

24. World Health Organization. Development of a WHO growth reference for school-aged children and adolescents. Bull World Health Organ. 2007;85:660-7.

25. Slaughter MH, Lohman TG, Boileau R, et al. Skinfold equations for estimation of body fatness in children and youth. Hum Biol. 1988;60:709-23.

26. Lohman TG. The use of skinfolds to estimate body fatness on children and youth. J Phys Educ Recreat Dance. 1987;58:98-103.

27. Gomes R, Silva L. Desordens alimentares e perfeccionismo: um estudo com atletas portugueses. Psicol Rev. 2010;16: 469-89.

28. Krentz EM, P Warschburger. Sports-related correlates of disordered eating in aesthetic sports. Psychol Sport Exerc. 2011;44:315-21.

29. Vieira JLL, Oliveira LP, Vieira LF, Vissoci JRN, Hoshino EF, Fernandes SL. Distúrbios de atitudes alimentares e sua relação com a distorção da auto-imagem corporal em atletas de judô do estado do Paraná. Rev Educ Fis/UEM. 2006; $17: 177-84$

\begin{tabular}{r|l} 
ENDEREço & \\
Leonardo de Sousa Fortes & Recebido para publicação: 27/10/2012 \\
R. Clóvis Bevilaqua, 163/1003 & Revisado: 07/07/14 \\
50710-330 - Recife - PE - BRASIL & Aceito: 13/10/2014 \\
e-mail: leodesousafortes@hotmail.com & \\
&
\end{tabular}

266 • Rev Bras Educ Fís Esporte, (São Paulo) 2015 Abr-Jun; 29(2):259-66 